\title{
Nota Científica \\ Propriedades físicas e energéticas da madeira e do carvão vegetal da espécie Hieronyma alchorneoides
}

\begin{abstract}
Adriel Furtado de Carvalho", Martha Andreia Brand ${ }^{1}$, Daniela Letícia Nones ${ }^{1}$, Franchesco Thomas de Marco ${ }^{1}$, Gustavo Friederichs ${ }^{1}$, Solange Maria Krug Weise ${ }^{2}$
\end{abstract}

${ }^{1}$ Universidade do Estado de Santa Catarina, n² 2090, Avenida Luiz de Camões, CEP 88520-000, Lages, SC, Brasil

²Universidade Regional de Blumenau, Campus 2 - Sala H-013, Rua São Paulo, n 3250, Itoupava Seca, Blumenau, SC, Brasil

\author{
"Autor correspondente: \\ adrielfurtado@yahoo.com.br \\ Termos para indexação: \\ Pirólise \\ Rendimento gravimétrico \\ Poder calorífico \\ Index terms: \\ Pyrolysis \\ Gravimetric yield \\ Calorific value
}

Histórico do artigo:

Recebido em 09/02/2014

Aprovado em 19/09/2014

Publicado em 10/10/2014

doi: 10.4336/2014.pfb.34.79.662

\begin{abstract}
Resumo - O objetivo deste trabalho foi determinar as propriedades físicas e energéticas da madeira e do carvão obtido pela carbonização da espécie Hieronyma alchorneoides Allemão (licurana). O carvão produzido na Agricultura Familiar de Biguaçu, SC, tem por finalidade a complementação renda. Destacam-se nas análises: a massa específica básica da madeira que apresentou $530 \mathrm{Kg} \mathrm{m}^{-3}$ e o poder calorífico superior de 4.315 $\mathrm{kcal} \mathrm{kg}^{-1}$. O rendimento gravimétrico foi de $37 \%$ e o poder calorífico superior de 6.740 $\mathrm{kcal} \mathrm{kg}^{-1}$. As propriedades físicas da madeira indicam aptidão da espécie para produção de carvão. A análise do carvão apresentou um ótimo rendimento gravimétrico.
\end{abstract}

\section{Physical and energetic properties of wood and charcoal of Hieronyma alchorneoides}

\begin{abstract}
The aim of this study was to determine the physical and energetic properties of wood and charcoal obtained by carbonization of Hieronyma alchorneoides Allemão (licurana). Coal produced in family farming Biguaçu, SC, is to supplement the income. The most important results are: specific gravity of the timber presented $530 \mathrm{Kg} \mathrm{m}^{-3}$ and the gross calorific value greater of $4.315 \mathrm{kcal} \mathrm{kg}^{-1}$. The coal gravimetric yield was $37 \%$ and gross calorific value was $6.740 \mathrm{kcal} \mathrm{kg}^{-1}$. The wood of $\mathrm{H}$. alchorneoides presents physical properties suitable for charcoal production. The charcoal showed excellent gravimetric yield.
\end{abstract}

Na microbacia de São Mateus, localidade de do Município de Biguaçu, SC, verifica-se que predominam pequenas unidades produtivas, onde em seu maior número a mão-de-obra é exclusivamente familiar, as quais sobrevivem da produção de aipim, banana, feijão, milho, batata-doce, hortaliças, laranja, café, batatainglesa, amendoim e cana-de-açúcar (Bauer, 2012).

Além disso, os agricultores familiares destas comunidades utilizam como complementação de renda, os recursos energéticos oriundos das formações secundárias da Floresta Ombrófila Densa para produção de carvão vegetal destinado ao uso doméstico (churrasco), que é comercializado no mercado regional (Carvalho, 2013).

Entrevistas realizadas com agricultores na região de Biguaçu, SC apontam a H. alchorneoides como sendo uma das "madeiras duras" mais citadas para a produção de carvão vegetal (Carvalho, 2013). A H. alchorneoides, 
está entre as 13 espécies nativas mais citadas segundo Justen et al. (2012), como de uso atual ou potencial de uso futuro no Estado de Santa Catarina.

Esta espécie pertence à família Phyllanthaceae, ela é abundante nos estágios sucessionais de capoeiras e capoeirões, onde pode tornar-se uma das dominantes, em terrenos abandonados com 30 a 50 anos, sobretudo em SC e PR (Lorenzi, 2002).

Trabalhos que caracterizem e avaliem a qualidade do carvão, produzido a partir das principais espécies utilizadas para a produção de carvão vegetal na Agricultura Familiar, são de suma importância, pois permitirão que os agricultores melhorem os processos produtivos dando qualidade ao produto final, valorizando o carvão produzido.

Desta forma, este trabalho teve o objetivo de avaliar as propriedades físicas e energéticas da madeira e do carvão vegetal produzido pela espécie florestal $H$. alchorneoides.

Para este estudo foram coletadas cinco árvores de $H$. alchorneoides, com idade inferior a 10 anos, na comunidade de São Mateus, no Município de Biguaçu, SC.

Do lote de madeira que seria carbonizada, duas árvores já estavam derrubadas e passaram por um período de secagem antes da coleta. Uma encontrava-se estocada exposta ao ambiente e a segunda sob cobertura, ambas por um período de duas a três semanas. De cada tora, que tinha em torno de $1 \mathrm{~m}$ de comprimento, foram descartadas as extremidades, aproximadamente $30 \mathrm{~cm}$ de cada ponta até a porção central. Da porção central foram serrados nove discos de $3 \mathrm{~cm}$ de espessura para a confecção dos corpos de prova e posteriores análises de laboratório.

Os outros três indivíduos foram obtidos a partir do corte de indivíduos em ambiente natural, em remanescente florestal. Destas foram retirados nove discos de $3 \mathrm{~cm}$ de espessura, a partir da altura do peito (DAP) para confecção dos corpos de prova. Neste estudo não foi avaliada a influência do diâmetro da tora (peça) na qualidade do carvão ou no processo de carbonização.

De todos os indivíduos amostrados, tanto os que se encontravam nas pilhas para secagem quanto os que estavam em área de floresta natural, foram utilizados três discos inteiros para determinação do teor de umidade na base úmida, conforme NBR 14929 (Associação Brasileira de Normas Técnicas, 2003a) e três discos inteiros para a determinação da densidade básica, conforme NBR 11491 (Associação Brasileira de Normas Técnicas, 2003b). O restante do material foi acondicionado em câmara climatizada para estabilização da umidade.

Para análise das propriedades físicas e energéticas do carvão foi realizado o procedimento de carbonização da madeira em laboratório. Para tanto, foram confeccionados sete corpos de prova (sub amostras) a partir dos discos obtidos de cada árvore, com dimensões aproximadas de $2 \mathrm{~cm} \times 2 \mathrm{~cm} \times 2 \mathrm{~cm}$. As amostras foram pesadas e medidas antes e após a carbonização, para a determinação da densidade aparente e do rendimento gravimétrico e volumétrico do carvão. Três corpos de prova, além dos utilizados para carbonização, foram selecionados para a determinação do teor de umidade antes da carbonização com as amostras previamente climatizadas para estabilização das mesmas.

Posteriormente, os corpos de prova foram envolvidos com papel alumínio, identificados, e colocados em mufla, para carbonização, conforme rampa de aquecimento, utilizada em trabalhos anteriores destinados a análise da estrutura anatômica do carvão vegetal (Muñiz, 2012; Nisgoski et al., 2012). Neste estudo não foi avaliada a influência da taxa de aquecimento na qualidade do carvão ou da umidade da madeira no processo de carbonização. A rampa de aquecimento pode ser visualizada na Tabela 1.

Tabela 1. Parâmetros utilizados para carbonização da madeira de Hyeronima alchorneoides.

\begin{tabular}{lcc}
\hline $\begin{array}{c}\text { Tempo } \\
(\mathbf{m i n})\end{array}$ & $\begin{array}{c}\text { Temperatura } \\
\left({ }^{\mathbf{O}} \mathbf{C}\right)\end{array}$ & $\begin{array}{c}\text { Taxa de } \\
\text { aquecimento } \\
\left({ }^{\circ} \mathbf{C} . \mathbf{m i n}^{-1}\right)\end{array}$ \\
\hline 00 & 25 & \\
\hline 20 & 150 & 7,50 \\
$87(1: 27 \mathrm{~h})$ & 200 & 2,30 \\
$184(3: 04 \mathrm{~h})$ & 250 & 1,36 \\
\hline $288(4: 48 \mathrm{~h})$ & 350 & 1,22 \\
\hline $362(6: 02 \mathrm{~h})$ & 450 & 1,24 \\
\hline $394(6: 32 \mathrm{~h})$ & 450 & \\
\hline
\end{tabular}

O rendimento gravimétrico foi determinado a partir da relação entre a massa seca do carvão e a massa seca da madeira, antes da carbonização. $\mathrm{O}$ rendimento volumétrico foi determinado a partir da relação entre o peso seco do carvão e o volume da madeira antes da carbonização (Brito \& Barrichelo, 1977). 
A análise energética da madeira e do material cabonizado (carvão) consistiu da determinação das propriedades de: teor de cinzas $\left(700^{\circ} \mathrm{C}\right)$, porcentagem de carbono fixo e porcentagem de voláteis $\left(900{ }^{\circ} \mathrm{C}\right)$ em termobalança gravimétrica (TGA), conforme a norma ASTM 1762 (American Society for Testing and Materials, 2007). O poder calorífico superior (PCS) foi determinado em calorímetro, conforme a norma DIN 51900 (Deutsches Institut für Normung, 2000) e o poder calorífico inferior (PCI), através de equação matemática descrita por Brand (2010). Também foi analisado a partir do material carbonizado o teor de umidade na base úmida segundo NBR 14929 (Associação Brasileira de Normas Técnicas, 2003b).

Na Tabela 2 são apresentados os valores médios das propriedades físicas e energéticas analisadas a partir da madeira de $H$. alchorneoides.

Houve variação significativa para o teor de umidade na base úmida. O indivíduo 1 estava estocado na pilha de toras ao lado do forno a céu aberto sofrendo ação do clima e o indivíduo 2, que também estava estocado, porém sob cobertura, dentro de um galpão, apresentaram menores teores de umidade, $34 \%$ e $37 \%$ respectivamente. Já os indivíduos $3(45 \%)$, 4 (48\%) e $5(61 \%)$ foram derrubados no momento da coleta, mantendo a sua umidade natural e por isso com umidade superior aos indivíduos 1 e 2. O teor de umidade ótimo para a carbonização da madeira encontra-se abaixo de $20 \%$ ou $30 \%$ da base seca (Valente, 1986), que corresponde a $25 \%$ na base úmida (Brand, 2010).

A massa específica básica média de $530 \mathrm{~kg} \mathrm{~m}^{-3}$ encontrada neste trabalho para $H$. alchorneoides, demonstra o excelente potencial energético desta espécie para a produção de carvão. Esta média foi semelhante à determinada para a Miconia cinnamomifolia $\left(552 \mathrm{~kg} \mathrm{~m}^{-3}\right)$, espécie também usada para a carbonização nos fornos de alvenaria nas propriedades familiares de Biguaçu, SC (Brand et al., 2013).

No entanto, o poder calorífico superior médio da madeira de $H$. alchorneoides $\left(4.315 \mathrm{kcal} \mathrm{kg}^{-1}\right)$ é mais elevado que o de $M$. cinnamomifolia $-4.178 \mathrm{kcal} \mathrm{kg}^{-1}$ (Brand et al., 2013).

A análise imediata da madeira revelou que o teor de voláteis médio $(79,39 \%)$ foi semelhante ao de $M$. cinnamomifolia $(80,17 \%)$ (Brand et al., 2013). O teor de cinzas médio foi inferior a $5 \%(0,78 \%)$, o que é positivo para o uso energético da madeira, tanto na forma de lenha como para a produção de carvão vegetal. O teor de carbono fixo médio para a espécie foi de 19,84\%. Brito \& Barrichelo (1982) preconizam que o teor de carbono fixo na madeira deve estar entre $15 \%$ a $25 \%$. Portanto o valor determinado para esta espécie está dentro dos intervalos definidos por estes autores.

As Tabelas 3 e 4 apresentam os dados relativos às propriedades físicas e energéticas do carvão de Hyeronima alchorneoides.

Considerando que a madeira neste estudo foi carbonizada com teor de umidade na base úmida de $12 \%$ $(\mathrm{RG}=37 \%) ; 11 \%(\mathrm{RG}=35 \%) ; 17 \%(\mathrm{RG}=37 \%) ; 16 \%$ $(\mathrm{RG}=39 \%)$ e $13 \%(\mathrm{RG}=39 \%)$, os valores de rendimento gravimétrico são semelhantes ao da $M$. cinnamomifolia que para teores de umidade $15 \%, 10 \%, 14 \%$ e $14 \%$, apresentaram os rendimentos gravimétricos de $36 \%$, $36 \%, 37 \%$ e 34\%, respectivamente (Brand et al., 2013).

Tabela 2. Propriedades físicas e energéticas da madeira de H. alchorneoides.

\begin{tabular}{cccccccc}
\hline Árvore & $\begin{array}{c}\text { TU rc } \\
(\%)\end{array}$ & $\begin{array}{c}\text { MEB } \\
\left(\mathbf{k g ~ m}^{-3}\right)\end{array}$ & $\begin{array}{c}\text { PCS } \\
\left(\mathbf{K c a l ~ k g}^{-1}\right)\end{array}$ & $\begin{array}{c}\text { PCI } \\
\left(\mathbf{k c a l ~ k g}^{-1}\right)\end{array}$ & $\begin{array}{c}\text { TV } \\
\mathbf{( \% )}\end{array}$ & $\begin{array}{c}\text { TC } \\
(\%)\end{array}$ & $\begin{array}{c}\mathbf{C F} \\
(\%)\end{array}$ \\
\hline 1 & $34 \mathbf{e}$ & $739 \mathbf{a}$ & $4.299 \mathbf{b c}$ & $3.975 \mathbf{b c}$ & $78,85 \mathbf{b}$ & $0,59 \mathbf{c}$ & $20,56 \mathbf{a}$ \\
2 & $37 \mathbf{d}$ & $438 \mathbf{d}$ & $4.249 \mathbf{c}$ & $3.925 \mathbf{c}$ & $80,40 \mathbf{a}$ & $0,79 \mathbf{b}$ & $18,81 \mathbf{c}$ \\
3 & $45 \mathbf{c}$ & $546 \mathbf{b}$ & $4.369 \mathbf{a}$ & $4.045 \mathbf{a b}$ & $79,08 \mathbf{a b}$ & $0,62 \mathbf{c}$ & $20,31 \mathbf{a b}$ \\
4 & $48 \mathbf{b}$ & $514 \mathbf{c}$ & $4.309 \mathbf{b}$ & $3.985 \mathbf{a b c}$ & $80,27 \mathbf{a}$ & $0,71 \mathbf{b c}$ & $19,02 \mathbf{b c}$ \\
5 & $61 \mathbf{a}$ & $412 \mathbf{e}$ & $4.348 \mathbf{a b}$ & $4.024 \mathbf{a}$ & $78,34 \mathbf{b}$ & $1,18 \mathbf{a}$ & $20,48 \mathbf{a}$ \\
\hline Média & 45 & 530 & 4.315 & 3.991 & 79,39 & 0,78 & 19,84 \\
\hline Desvio padrão & 10,607 & 129,016 & 46,510 & 46,510 & 0,906 & 0,238 & 0,849 \\
\hline
\end{tabular}

$\mathrm{TU}$ rc $=$ teor de umidade recém chegado - base úmida; $\mathrm{MEB}=$ massa específica básica; $\mathrm{PCS}=$ poder calorífico superior; $\mathrm{PCI}=$ poder calorífico inferior; $\mathrm{TV}=$ teor de voláteis; $\mathrm{TC}=$ teor de cinzas; $\mathrm{CF}=$ teor de carbono fixo. Médias seguidas da mesma letra, na coluna, não apresentam diferença estatística significativa para o teste de Tukey $(\mathrm{P}>0,05)$. 
Tabela 3. Propriedades físicas do carvão de Hyeronima alchorneoides.

\begin{tabular}{ccccccccc}
\hline Árvore & $\begin{array}{c}\text { TU mad. } \\
(\%)\end{array}$ & RG (\%) & RV (\%) & $\begin{array}{c}\text { TU car. } \\
(\%)\end{array}$ \\
\hline $\mathbf{1}$ & 12 & c & 37 & ab & 28 & a & 6 & a \\
$\mathbf{2}$ & 11 & c & 35 & b & 16 & cd & 6 & a \\
$\mathbf{3}$ & 17 & a & 37 & ab & 20 & b & 5 & b \\
\hline $\mathbf{4}$ & 16 & a & 39 & a & 19 & bc & 5 & b \\
$\mathbf{5}$ & 13 & b & 39 & ab & 15 & d & 5 & ab \\
\hline Média & $\mathbf{1 4}$ & \multicolumn{3}{c}{$\mathbf{3 7}$} & $\mathbf{2 0}$ & $\mathbf{5 , 4}$ \\
\hline
\end{tabular}

$\mathrm{TU}$ mad. = teor de umidade da madeira - base úmida; $\mathrm{TU}$ car. $=$ teor de umidade do carvão; $\mathrm{RG}$ = rendimento gravimétrico do carvão; $\mathrm{RV}=$ rendimento volumétrico do carvão. Médias seguidas da mesma letra, na coluna, não apresentam diferença estatística significativa para o teste de Tukey $(\mathrm{P}>0,05)$.

De forma geral as amostras apresentaram baixa umidade. Ainda assim, ocorreu variação estatística significativa para o teor de umidade do carvão entre os indivíduos. Porém, estas diferenças não excederam $2 \%$ de umidade.

O poder calorífico médio do carvão da espécie foi de $6.740 \mathrm{kcal} \mathrm{kg}^{-1}$, não havendo diferença significativa entre as árvores analisadas. Este valor foi superior aos encontrados por Brand et al. (2013) para a $M$. cinnamomifolia (6.267 kcal kg-1).

A análise imediata do carvão produzido com $H$. alchorneoides apresentou 30,78\% de teor médio de materiais voláteis. $\mathrm{O}$ carvão vegetal com elevado teor de voláteis produzido no sistema artesanal (processo de carbonização não controlado) pode ser prejudicial à saúde, pois estas substâncias são liberadas diretamente no alimento (Rosa, 2010).

A média de teor de cinzas encontrada no carvão (3,04\%), foi superior ao determinado por Brand et al. (2013) para o carvão de M. cinnamomifolia $(1,35 \%)$. Por outro lado, à média obtida para carbono fixo $(66,36 \%)$ foi inferior ao da $M$. cinnamomifolia $(68,18 \%)$, apesar de muito próximo.

Este trabalho mostrou que a massa específica básica da madeira de $H$. alchorneoides, apresenta excelente potencial energético. Em relação ao carvão produzido pela espécie, o teor de umidade baixo e o elevado rendimento gravimétrico, são características positivas para um produto qualidade.

Tabela 4. Propriedades energéticas do carvão de Hyeronima alchorneiodes.

\begin{tabular}{cccccccccccc}
\hline Árvore & $\begin{array}{c}\text { MEA } \\
\left(\mathbf{k g ~ m}^{-3}\right)\end{array}$ & \multicolumn{2}{c}{$\begin{array}{c}\text { PCS } \\
\left(\mathbf{k c a l ~ k g}^{-1}\right)\end{array}$} & $\begin{array}{c}\text { PCI } \\
\left(\mathbf{k c a l ~ k g}^{-1}\right)\end{array}$ & $\begin{array}{c}\text { TV } \\
(\mathbf{\%})\end{array}$ & $\begin{array}{c}\text { TC } \\
\mathbf{( \% )}\end{array}$ & $\begin{array}{c}\mathbf{C F} \\
(\mathbf{\%})\end{array}$ \\
\hline $\mathbf{1}$ & 445 & $\mathbf{a}$ & 6.742 & $\mathbf{a}$ & $6.418 \mathbf{a}$ & 28,98 & $\mathbf{b}$ & 1,30 & $\mathbf{c}$ & 69,72 & $\mathbf{a}$ \\
$\mathbf{2}$ & 308 & $\mathbf{b}$ & 6.850 & $\mathbf{a}$ & $6.526 \mathbf{a}$ & 27,51 & $\mathbf{b}$ & 1,46 & $\mathbf{c}$ & 71,03 & $\mathbf{a}$ \\
$\mathbf{3}$ & 280 & $\mathbf{b c}$ & 6.654 & $\mathbf{a}$ & $6.330 \mathbf{a}$ & 34,65 & $\mathbf{a}$ & 4,71 & $\mathbf{a}$ & 60,64 & $\mathbf{c}$ \\
$\mathbf{4}$ & 264 & $\mathbf{b c}$ & 6.649 & $\mathbf{a}$ & $6.325 \mathbf{a}$ & 31,58 & $\mathbf{a b}$ & 4,83 & $\mathbf{a}$ & 63,59 & $\mathbf{b c}$ \\
$\mathbf{5}$ & 234 & $\mathbf{c}$ & 6.808 & $\mathbf{a}$ & $6.484 \mathbf{a}$ & 30,22 & $\mathbf{a b}$ & 2,96 & $\mathbf{b}$ & 66,82 & $\mathbf{a b}$ \\
\hline Média & $\mathbf{3 2 5}$ & & $\mathbf{6 . 7 4 0}$ & & $\mathbf{6 . 4 1 6}$ & $\mathbf{3 0 , 7 8}$ & $\mathbf{3 , 0 4}$ & & $\mathbf{6 6 , 3 6}$ \\
\hline
\end{tabular}

MEA = massa específica aparente do carvão; $\mathrm{PCS}=$ poder calorífico superior; $\mathrm{PCI}=$ poder calorífico inferior; $\mathrm{TV}=$ teor de voláteis; $\mathrm{TC}=$ teor de cinzas; $\mathrm{CF}=$ carbono fixo. Médias seguidas da mesma letra, na coluna, não apresentam diferença estatística significativa para o teste de Tukey $(\mathrm{P}>.0,05)$.

\section{Referências}

AMERICAN SOCIETY FOR TESTING AND MATERIALS. D-1762: Standard Test Method for Chemical Analysis of wood charcoal. Philadelphia, 2007.

ASSOCIAÇÃO BRASILEIRA DE NORMAS TÉCNICAS. NBR 14929: madeira - determinação do teor de umidade de cavacos método por secagem em estufa. Rio de Janeiro, 2003a.

ASSOCIAÇÃO BRASILEIRA DE NORMAS TÉCNICAS. NBR 11491: determinação da densidade básica. Rio de Janeiro, 2003b.

BAUER, E. Mudanças no uso da terra em Biguaçu - SC: agricultores em permanente processo de adaptação. 2012. $94 \mathrm{f}$. Dissertação (Mestrado em Agroecossistemas) - Universidade Federal de Santa Catarina, Florianópolis.
BRAND, M. A.; CUNHA A. B.; CARVALHO, A. F.; BREHMER D. R.; KÜSTER L. C. Análise da qualidade da madeira e do carvão vegetal produzido à partir da espécie Miconia cinnamomifolia (De Candolle) Naudin (Jacatirão-açu) na agricultura familiar, em Biguaçu, Santa Catarina. Scientia Forestalis, Piracicaba. v. 41, n. 99, p. 401-410, 2013.

BRAND, M. A. Energia de biomassa florestal. Rio de Janeiro: Interciência, 2010. 114 p.

BRITO, J. O.; BARRICHELO, L. E. G. Aspectos técnicos da utilização da madeira e carvão vegetal como combustíveis. In: SEMINÁRIO DE ABASTECIMENTOENERGÉTICO INDUSTRIALCOMRECURSOS FLORESTAIS, 2., 1982, São Paulo. Palestras apresentadas... São Paulo: Coordenadoria da Indústria e Comércio, 1982. p. 101-137. 
BRITO, J. O.; BARRICHELO, L. E. G. Correlações entre características físicas e químicas da madeira e a produção de carvão vegetal: i. Densidade e teor de lignina da madeira de eucalipto. IPEF, Piracicaba, v. 14, p. 9-20, 1977.

CARVALHO, A. F. Caracterização da madeira e do carvão vegetal produzido a partir de cinco espécies florestais utilizadas na região de Biguaçu, SC. 2013. 142 f. Dissertação (Mestrado em Engenharia Florestal), Universidade do Estado de Santa Catarina, Lages.

DEUTSCHES INSTITUT FÜR NORMUNG. DIN 51900: determining the gross calorific value of solid and liquid fuels using the bomb calorimeter, and calculation of net calorific value. Berlim, 2000.

JUSTEN, J. G. K.; MÜLLER, J. J. V.; TORESAN, L. Levantamento Socioambiental. In: VIBRANS, A. C.; SEVEGNANI, L.; GASPER, A. L. de; LINGNER, D. V. (Ed.). Inventário florístico florestal de Santa Catarina. Blumenau: EDIFURB, 2012.v 1. Título do volume 1: Diversidade e conservação dos remanescentes florestais.
LORENZI, H. Árvores brasileiras: manual de identificação e cultivos de plantas arbóreas do Brasil. 2. ed. São Paulo: Nova Odessa, 2002.

MUÑIZ, G. I. B.; NISGOSKI, S.; SHARDOSIN, F. Z.; FRANÇA, R. F.. Anatomia do carvão de espécies florestais. Cerne, Lavras, v. 18, n. 3, p. 471-477, 2012.

NISGOSKI, S.; BOLZON MUÑIZ, G. I.; FRANÇA, R. F.; BATISTA, F. R. R. Anatomia do lenho carbonizado de Copaifera $c f$. langsdorfii Desf. E Dipteryx odorata (Aubl.) Wild. Revista Ciência da Madeira, Pelotas, v. 3, n. 2, 2012.

ROSA, R. F. Qualidade do carvão vegetal, para uso doméstico, comercializado em três municípios do estado do Espírito Santo. 2010. 55 f. Monografia (Graduação em Engenharia Industrial Madeireira), Universidade Federal do Espírito Santo, Jerônimo Monteiro.

VALENTE, O. F. Carbonização da madeira de eucalipto. Informe Agropecuário. Belo Horizonte, v. 141, p. 74-79, 1986. 